\title{
Changes in sebum levels and the development of acneiform rash in patients with non-small cell lung cancer after treatment with EGFR inhibitors
}

This article was published in the following Dove Press journal:

OncoTargets and Therapy

28 January 2015

Number of times this article has been viewed

\author{
Takeshi Nakahara ${ }^{1,2}$ \\ Yoichi Moroi ${ }^{2}$ \\ Koichi Takayama ${ }^{3}$ \\ Eriko Itoh ${ }^{1,2}$ \\ Makiko Kido-Nakahara ${ }^{2}$ \\ Yoichi Nakanishi ${ }^{3}$ \\ Masutaka Furue ${ }^{2}$ \\ 'Division of Skin Surface Sensing, \\ ${ }^{2}$ Department of Dermatology, \\ ${ }^{3}$ Research Institute for Diseases \\ of the Chest, Graduate School \\ of Medical Sciences, Kyushu \\ University, Fukuoka, Japan
}

Background: It has recently been shown that patients treated with epidermal growth factor receptor (EGFR) inhibitors often develop various cutaneous adverse events. While the pathogenesis underlying these events remains unclear, the relationship between skin toxicity induced by EGFR inhibitors and the sebaceous glands that express EGFR has been previously reported.

Objectives: The primary aim of this study was to determine the relationship between cutaneous sebum levels and acneiform rash, a typical skin toxicity of EGFR inhibitors, by measuring the sebum levels before and after EGFR inhibitor treatment.

Methods: Eight patients diagnosed with non-small cell lung cancer (NSCLC) (three men and five women with an average age of 69.3 years) who were initiated on treatment with EGFR inhibitors (either gefitinib [Iressa $\left.{ }^{\circledR}\right]$ or erlotinib $\left[\right.$ Tarceva $\left.^{\circledR}\right]$ ) were enrolled. Using a Sebumeter ${ }^{\mathbb{B}}$, sebum levels in the face, chest, and back of each patient were measured before and after EGFR inhibitor treatment. The development of acneiform rash in each skin region was also assessed.

Results: Changes in sebum level along with the development of an acneiform rash were observed after patients were started on EGFR inhibitor treatment. Patients who developed an EGFR inhibitor-induced acneiform rash tended to have higher pretreatment sebum levels (baseline) than did patients who did not experience an acneiform rash. At each time point measurement, sebum levels were found to be significantly higher in patients who had developed an acneiform rash at that time. Patients who developed rash during treatment showed greater differences in sebum level compared with pretreatment baseline.

Conclusion: Patients who had increased levels of sebum or whose sebum levels showed greater change from pretreatment baseline developed an acneiform rash, suggesting that sebaceous gland activity may be involved in the mechanism underlying the development of acneiform rash, in patients treated with EGFR inhibitors.

Keywords: skin toxicity, cutaneous adverse event, gefitinib, erlotinib

\section{Introduction}

There has been an increasing use of epidermal growth factor receptor (EGFR) inhibitors, and their efficacy has been demonstrated in patients treated for advanced non-small cell lung cancer (NSCLC). ${ }^{1}$ However, patients treated with EGFR inhibitors are frequently known to develop skin toxicity, which is associated with reduced quality of life in patients. ${ }^{2}$ Interestingly, some studies have reported a positive correlation between skin toxicity and treatment response, thus highlighting the importance of managing skin toxicity in order to enable continued treatment..$^{3-5}$ Little is known, however, regarding the mechanism underlying EGFR inhibitor-induced skin toxicity, which first manifests as a form of acneiform rash immediately after treatment is initiated. It is established that EGFR is expressed in the sebaceous glands, ${ }^{2}$ and a correlation between EGFR inhibitor
Correspondence: Takeshi Nakahara Division of Skin Surface Sensing, Graduate School of Medical Sciences, Kyushu University, Maidashi 3-I-I Higashiku, Fukuoka, 812 8582, Japan $\mathrm{Tel}+8 \mathrm{I} 926425585$

Fax+8I 926425600

Email nakahara@dermatol.med.kyushu-u. ac.jp 
use and changes to sebaceous glands has been reported. ${ }^{6}$ The aim of this study was to demonstrate the relationship between sebum and acneiform rash by measuring sebum levels and their change before and after EGFR inhibitor treatment.

\section{Patients and methods}

After obtaining approval from the Kyushu University Hospital ethics committee, eight consenting patients with confirmed NSCLC (three men and five women with an average age of 69.3 years [age range 57-84 years]) for whom gefitinib (Iressa ${ }^{\circledR}$ ) or erlotinib (Tarceva ${ }^{\circledR}$ ) was indicated were enrolled.

Sebum levels were measured periodically (ie, at baseline and at 1, 2, 3, 4, and 6 weeks after the initiation of EGFR inhibitor therapy) on skin regions of approximately $3 \times 3 \mathrm{~cm}$, in the shape of a square, at three different sites (the face, the chest, and the back), using a Sebumeter ${ }^{\circledR}$ SM815 (Courage+Khazaka Electronic GmbH, Cologne, Germany). Sebum was collected at four different locations in each skin region, and the average sebum level was calculated from the four measurements for each site. For the face, measurements were taken from three different regions (ie, forehead and right and left cheeks), and the average facial sebum value from the three measurements was obtained.
The acneiform rash in each skin region was also evaluated each time measurements were taken. The absolute value of the differences in sebum secretion compared with pretreatment baseline was calculated for each patient at each time point.

Statistical analysis was performed using the Mann-Whitney $U$-test (SPSS Version 18). The absolute values of sebum secretion for each time point, and the difference in sebum secretion values between each treatment time point and pretreatment baseline were categorized into two groups according to the presence or absence of rash. The average values were further calculated from all data points at each site (ie, face, chest, and back) and utilized for statistical analysis.

\section{Results}

The sebum levels increased transiently after initiation of treatment and then subsequently decreased. The rate at which acneiform rash developed also increased after the start of treatment and decreased, and then increased again, showing a wavelike fluctuation during the study period. The development of acneiform rash was accompanied by changes in sebum level, with both parameters showing similar patterns of change over time at some sites (Figure 1).
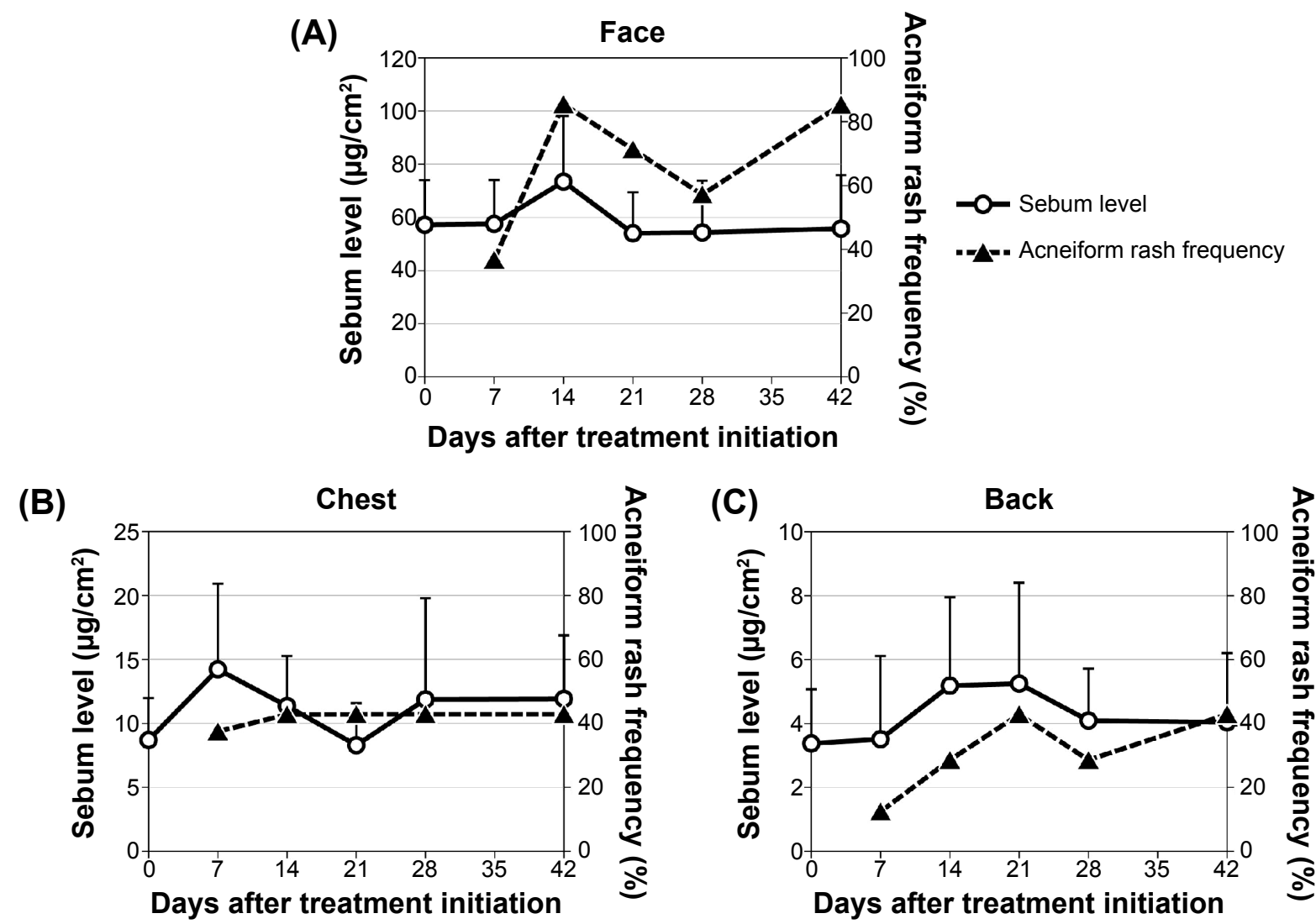

Figure I (A-C) Frequency of acneiform rash development in relation to sebum levels, following EGFR inhibitor treatment in NSCLC.

Notes: Average sebum level was calculated from seven or eight patients (expressed as mean + SE). Average acneiform rash frequency was calculated from seven or eight patients (expressed as mean).

Abbreviations: EGFR, epidermal growth factor receptor; NSCLC, non-small cell lung cancer. 
In order to determine the correlation between sebum levels or their change during treatment, and the manifestation of acneiform rash, the relationship between pretreatment sebum levels and the occurrence of acneiform rash at each time point (ie, 1, 2, 3, 4, and 6 weeks after initiation of treatment) was assessed for each site. High pretreatment sebum levels tended to be associated with the development of acneiform rash at each time point and at each site (Figure 2).

The correlation between sebum levels (at each time point) and the presence of acneiform rash at each time point (ie, 1 , 2, 3, 4, and 6 weeks after the initiation of treatment) were evaluated. Patients who developed acneiform rash had significantly higher sebum levels at each time point (Figure 3). Sebum levels were higher in the face compared with the chest or the back. However, acneiform rash appeared at a higher rate, not only in the face but also, in the chest and the back. The increased levels of sebum were also linked to the occurrence of acneiform rash in the chest and back (Figures 2 and 3 ). We then determined the differences in sebum levels compared with pretreatment baseline and examined their relation to the acneiform rash. Critically, greater changes in sebum levels from the start of the treatment were observed in patients who developed acneiform rash (Figure 4).

\section{Discussion}

EGFR is expressed in various tissues of normal skin, including the basal cells of the epidermis, the sebaceous glands, outer root sheath cells, the eccrine glands, and vascular smooth muscle cells. ${ }^{2}$ EGFR inhibitors are known to suppress proliferation and differentiation of these tissues. The involvement of EGFR in the pathology of acneiform rash includes affected sebaceous glands, keratin plug formation induced by hair dyskeratosis, and hair follicle inflammation. ${ }^{\text {? }}$ However, considering how the rash often develops during the early stages of EGFR inhibitor therapy, the direct effect of EGFR inhibitors on sebaceous glands seems to play an important role in the etiology of acneiform rash. ${ }^{6,7}$ Indeed, in the present study it was observed that the development of acneiform rash was accompanied by changes in sebum level; this observation was noted during the initial stages of treatment, with both parameters showing a similar pattern of change over time at some sites. It was also found that in patients who developed acneiform rash, the sebum levels both at pretreatment baseline and at each time point were higher than that in those who did not have acneiform rash, suggesting a correlation between sebum level and acneiform rash. However, it was noted that acneiform rash developed even on
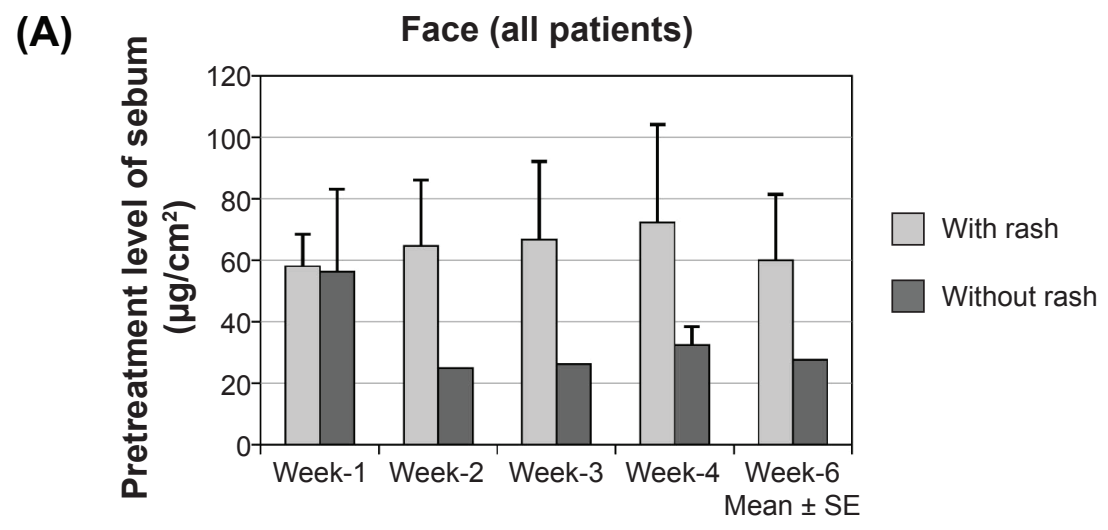

(B)

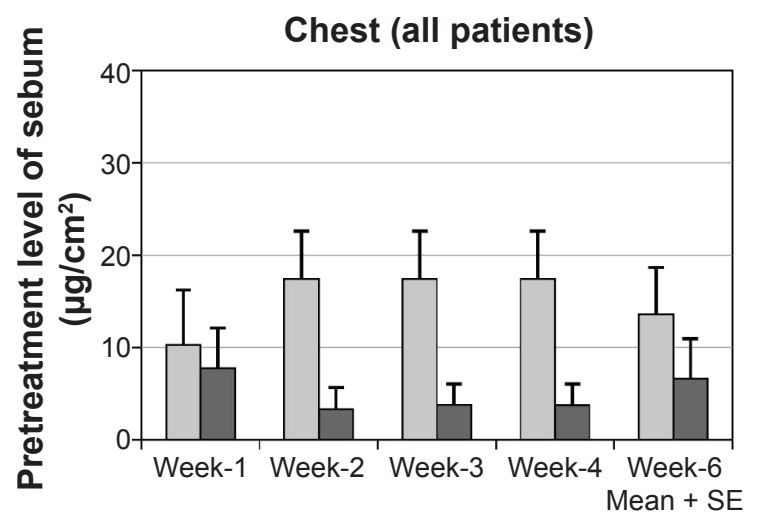

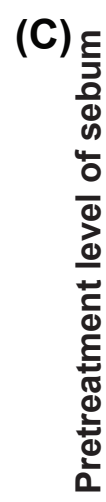

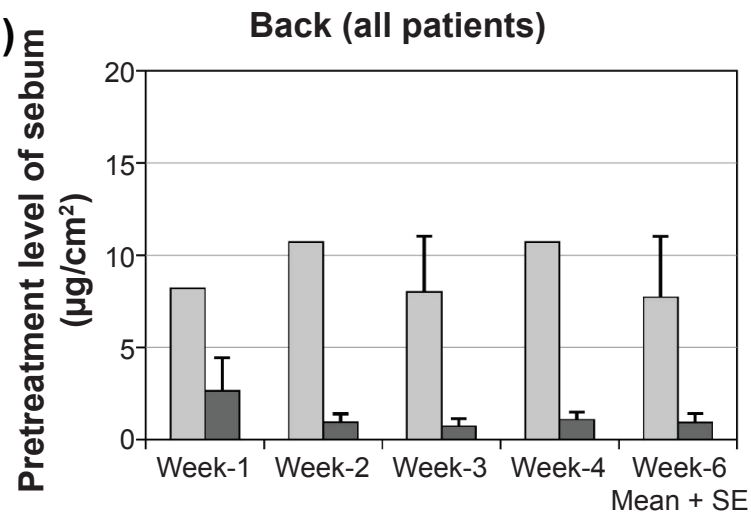

Figure 2 (A-C) Development of acneiform rash over time and pretreatment sebum levels at different body sites. 


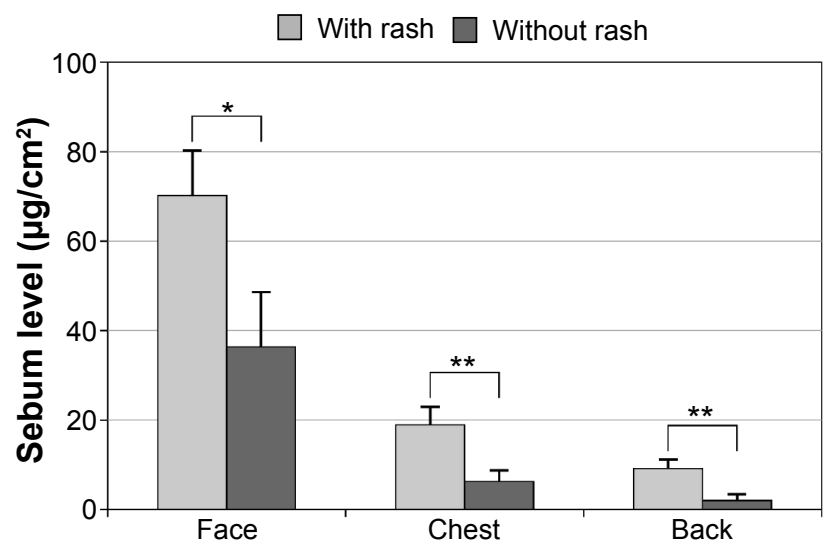

Figure 3 Presence of acneiform rash and sebum levels at each time point (calculated from all time points and all patients).

Notes: Values are expressed as mean \pm SE. $* P<0.05$; $* * P<0.0$ I (Mann-Whitney). Abbreviation: SE, standard error of the mean.

the chest and the back, where sebum levels are not as high as on the face. These results therefore strongly suggest that the correlation between sebum level and acneiform rash may be site-specific. The differences in sebum level as compared with pretreatment baseline also appeared to be associated with the development of acneiform rash. The magnitude of change in sebum levels as well as the amount of sebum secretion may contribute to EGFR inhibitor-induced acneiform eruption, suggesting an association between sebaceous gland activity and susceptibility to EGFR inhibitor-induced skin toxicity.

In a recent study, researchers observed elevated expression levels of various chemokines, followed by the infiltration of inflammatory cells, in a mouse model that lacked EGFR in the epidermis, demonstrating a possible mechanism for EGFR inhibitor-induced skin toxicity. ${ }^{8,9}$ From these reports, together with the present findings, a possible

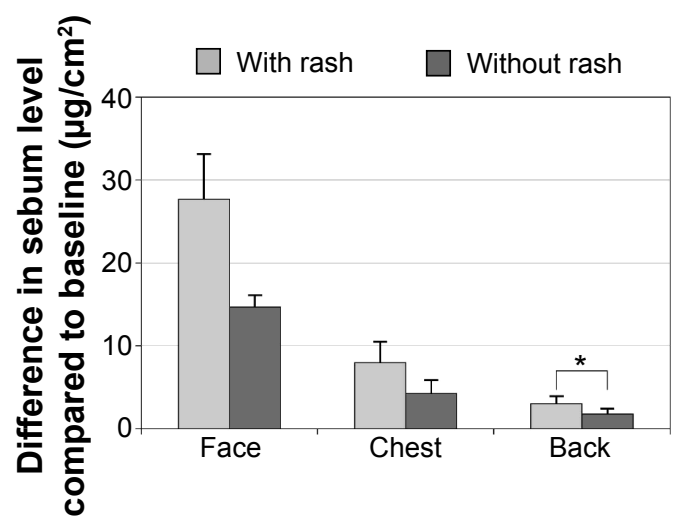

Figure 4 Presence of acneiform rash and differences in sebum level compared with pretreatment baseline, at each time point.

Notes: Calculated from all time points and all patients; values are expressed as mean \pm SE. $* P<0.05$ (Mann-Whitney).

Abbreviation: SE, standard error of the mean. mechanism by which patients develop acneiform rash can be postulated. The levels of sebaceous gland activity vary among individuals, and patients with higher levels of activity may have enhanced sensitivity to EGFR inhibitors, resulting in increased inflammatory cell infiltration in the sebaceous glands. Eventually, the inflammation in sebaceous glands establishes acneiform rash, characteristic morphological skin toxicity, and change in sebum levels. It was reported that inflammatory mediators, such as leukotriene B4 or prostaglandin J2, can increase sebum production. ${ }^{10,11}$ Spread of the inflammation to sebaceous glands might enhance sebum secretion, by inducing such inflammatory mediators. On the other hand, it is well known that patients treated with EGFRI develop progressive skin dryness, which is inconsistent with high sebum levels. Lichtenberger et al described changes in epidermal structure that were associated with decreased expression of the tight junction genes claudin 1 and claudin 3 as well as occludin and resulted in decreased water retention of the epidermis in EGFR-knockout mice. ${ }^{8}$ Mascia et al described alterations in keratinocyte differentiation marker expression. ${ }^{9}$ These studies and our study indicate that the effector sites of EGFR in acneiform rash and skin dryness might be quite different.

As patients with high sebum levels develop acneiform rash more frequently, medications effective in reducing sebum production, including retinoic acid (tretinoin) and adapalene, may be beneficial for treating acneiform rash. Taking into account other studies that have shown the correlation of EGFR inhibitor-induced skin toxicity and favorable treatment response, ${ }^{3-5}$ analyses of sebum secretion levels and their changes over time may therefore be useful in predicting clinical outcome.

\section{Disclosure}

This study was supported by an unrestricted educational grant from the nonprofit organization JASMIN, given to Kyushu University Hospital. The authors have no other conflicts of interest in this work.

\section{References}

1. Cufer T, Ovcaricek T, O'Brien ME. Systemic therapy of advanced nonsmall cell lung cancer: major-developments of the last 5-years. Eur J Cancer. 2013;49(6):1216-1225.

2. Lacouture ME. Mechanisms of cutaneous toxicities to EGFR inhibitors. Nat Rev Cancer. 2006;6(10):803-812.

3. Orditura M, De Vita F, Galizia G, et al. Correlation between efficacy and skin rash occurrence following treatment with the epidermal growth factor receptor inhibitor cetuximab: a single institution retrospective analysis. Oncol Rep. 2009;21(4):1023-1028.

4. Jonker DJ, O'Callaghan CJ, Karapetis CS, et al. Cetuximab for the treatment of colorectal cancer. N Engl J Med. 2007;357(20):2040-2048. 
5. Wacker B, Nagrani T, Weinberg J, Witt K, Clark G, Cagnoni PJ. Correlation between development of rash and efficacy in patients treated with the epidermal growth factor receptor tyrosine kinase inhibitor erlotinib in two large phase III studies. Clin Cancer Res. 2007;13(13):3913-3921.

6. Takata T, Tarutani M, Zouboulis CC, Sano S. Sebaceous glands as the primary target of EGFR-inhibitors in the development of papulopustular eruption. J Dermatol Sci. 2012;66(2):165-168.

7. Guttman-Yassky E, Mita A, De Jonge M, et al. Characterisation of the cutaneous pathology in non-small cell lung cancer (NSCLC) patients treated with the EGFR tyrosine kinase inhibitor erlotinib. Eur J Cancer. 2010;46(11):2010-2019.

8. Lichtenberger BM, Gerber PA, Holcmann M, et al. Epidermal EGFR controls cutaneous host defense and prevents inflammation. Sci Transl Med. 2013;5(199):199ra111.
9. Mascia F, Lam G, Keith C, et al. Genetic ablation of epidermal EGFR reveals the dynamic origin of adverse effects of anti-EGFR therapy. Sci Transl Med. 2013;5(199):199ra110.

10. Iwata C, Akimoto N, Sato T, Morokuma Y, Ito A. Augmentation of lipogenesis by 15-deoxy-Delta12,14-prostaglandin J2 in hamster sebaceous glands: identification of cytochrome P-450-mediated 15-deoxyDelta12,14-prostaglandin J2 production. J Invest Dermatol. 2005; 125(5):865-872.

11. Alestas T, Ganceviciene R, Fimmel S, Müller-Decker K, Zouboulis CC. Enzymes involved in the biosynthesis of leukotriene B4 and prostaglandin E2 are active in sebaceous glands. $J$ Mol Med (Berl). 2006; 84(1):75-87.

\section{Publish your work in this journal}

OncoTargets and Therapy is an international, peer-reviewed, open access journal focusing on the pathological basis of all cancers, potential targets for therapy and treatment protocols employed to improve the management of cancer patients. The journal also focuses on the impact of management programs and new therapeutic agents and protocols on

\section{Dovepress}

patient perspectives such as quality of life, adherence and satisfaction. The manuscript management system is completely online and includes a very quick and fair peer-review system, which is all easy to use. Visit http://www.dovepress.com/testimonials.php to read real quotes from published authors.

Submit your manuscript here: http://www.dovepress.com/oncotargets-and-therapy-journal 